\title{
Gute Kartographische Nachrichten
}

\section{Jochen Schiewe ${ }^{1}$}

Accepted: 15 February 2022 / Published online: 2 March 2022

(c) The Author(s) 2022, corrected publication 2022

Liebe Mitglieder,

Liebe Kartographiebegeisterte!

Das erste KN-Heft des Jahrganges 2022 können wir erfreulicherweise mit einer Reihe von guten Nachrichten beginnen.

Die bedeutendste Nachricht gleich vorneweg: Prof. Dr. Ulrich Freitag kam im Dezember gar nicht mehr aus dem Feiern heraus-erst beging er seinen 90. Geburtstag (siehe auch den Beitrag in KN 4/2021), dann erhielt er im Rahmen der International Cartographic Conference (ICC) in Florenz die Carl Mannerfelt Gold Medal. Mit dieser werden Kartographinnen und Kartographen mit außergewöhnlichen Verdiensten und Beiträgen für die internationale Kartographie geehrt. Die große Bedeutung dieser Würdigung wird dadurch deutlich, dass sie nur selten (zwischen 1979 und 2020 nur 13-mal) und nun das erste Mal überhaupt an einen Deutschen verliehen wird. Ohne die Laudatio auf Prof. Freitag zu wiederholen (diese finden Sie in diesem Heft): Zum einen ist sein vorbildliches Wirken als Hochschullehrer, Forscher, internationaler und nationaler Netzwerker und nicht zuletzt als ehrenamtlich Engagierter zu erwähnen (z. B. als mein Vor-vor-vor-vorgänger im Amt des DGfK-Präsidenten). Zum anderen möchte ich aber auch die außergewöhnlich angenehme Persönlichkeit von Prof. Freitag hervorheben —er hat einen Stil geprägt, der heute noch geschätzt wird und uns als Leitbild dient. Im Namen des Vorstandes-und ganz sicherlich auch im Namen aller Mitglieder der DGfK: Herzlichen Glückwunsch zu dieser besonderen Auszeichnung!

Bleiben wir bei der ICC in Florenz (zu der es in dieser Ausgabe auch einen ausführlicheren Bericht gibt). Nach langer Wartezeit gab es auch endlich wieder eine Auszeichnung für einen deutschen Beitrag im Rahmen der Kartenausstellung: Das Bundesamt für Kartographie und Geodäsie

Jochen Schiewe

jochen.schiewe@hcu-hamburg.de

1 HafenCity University Hamburg, Hamburg, Germany
(BKG) hat den Publikumspreis für die „Mini-map of the World 1: 100 Mio.“ in der Kategorie „Educational products“ gewonnen! Diese originelle Weltkarte auf Papier kommt in der Größe einer Kreditkarte daher und kann auf eine Größe von $40 \times 30 \mathrm{~cm}$ entfaltet werden. Herzlichen Glückwunsch $\mathrm{zu}$ diesem beeindruckenden Produkt und der verdienten Würdigung!

Und noch einmal ICC: Auch wenn es keine offizielle Ehrung gab, war die deutsche Teilnahme am Barbara-Petchenik-Kinderkartenwettbewerb 2021 mehr als lobenswert. Dank der Initiative unserer Vizepräsidentin Internationales, Anja Hopfstock, gab es eine rekordverdächtige Teilnahme von 42 Beiträgen, aus denen die Jury sechs Karten auswählte, die in Florenz präsentiert wurden. Sicherlich gibt es in den kommenden Wochen und Monaten noch Gelegenheiten, sich diese beeindruckenden Karten der Kinder und Jugendlichen im Original anzusehen-einen digitalen Eindruck gewinnen Sie bereits unter https://barbara-petch enik.dgfk.net/2021/09/26/herzlichen-glueckwunsch/. Allen Teilnehmerinnen und Teilnehmern und nationalen Gewinnerinnen und Gewinnern dieses Wettbewerbs: Herzlichen Glückwunsch!

Aber auch abseits der ICC gibt es gute Nachrichten: Zum zweiten Mal konnten wir die Nico Rüpke-Kartographiepreise vergeben. Dank der großzügigen Unterstützung der Nico Rüpke-Stiftung Hamburg ist es uns möglich, die besten wissenschaftlichen und praktischen Beiträge in den $\mathrm{KN}$, aber auch die besten Gutachter sowie weitere besonders engagierte Personen auszuzeichnen. Eine Übersicht der Preisträger finden Sie ebenfalls in dieser Ausgabe. Natürlich auch an dieser Stelle: Herzlichen Glückwunsch!

Mitte Januar trafen sich Vorstand, Vorstandsrat sowie einige weitere Personen erneut zu einem DGfK-Klausurtag. Mit dem Ziel, die DGfK für Mitglieder und Noch-nicht-Mitglieder attraktiver zu machen, wurde in vielen Kleingruppen diskutiert und wurden viele konkrete Ideen entwickelt. Einen ersten Bericht zum Klausurtag finden Sie ebenfalls in dieser $\mathrm{KN}$-Ausgabe, eine ausführliche Darstellung folgt in Heft 2/2022 (ganz sicherlich auch verbunden mit der Aufforderung zu Ihrer Mitwirkung!). 
Und schließlich gibt es auch noch die Vorfreude: Die Zeichen stehen nicht schlecht, dass wir uns im Sommer auch wieder in Präsenz treffen können. Zum einen werden wir im Juni einen Sommerempfang veranstalten, der den ausgefallenen Neujahrsempfang ersetzen soll. Zum anderen freuen wir uns auf den nächsten Deutschen Kartographie Kongress in Wien, der zusammen mit unseren Kolleginnen und Kollegen aus Österreich, der Schweiz und Großbritannien vom 19. bis zum 21. September stattfinden wird. Aktuelle Informationen hierzu erhalten Sie auf der Website https://eurocarto2022.org/.

Ich hoffe, ich konnte Ihnen mit diesem bunten Strauß an guten kartographischen Nachrichten Lust auf ein interessantes und freudiges Jahr 2022 machen. So kann es gerne weitergehen! Helfen Sie bitte dabei, dass wir auch in den kommenden Ausgaben viele positive Meldungen haben werden.

Ihr Jochen Schiewe
Supplementary Information The online version contains supplementary material available at https://doi.org/10.1007/s42489-022-00100-y.

Funding Open Access funding enabled and organized by Projekt DEAL.

Open Access This article is licensed under a Creative Commons Attribution 4.0 International License, which permits use, sharing, adaptation, distribution and reproduction in any medium or format, as long as you give appropriate credit to the original author(s) and the source, provide a link to the Creative Commons licence, and indicate if changes were made. The images or other third party material in this article are included in the article's Creative Commons licence, unless indicated otherwise in a credit line to the material. If material is not included in the article's Creative Commons licence and your intended use is not permitted by statutory regulation or exceeds the permitted use, you will need to obtain permission directly from the copyright holder. To view a copy of this licence, visit http://creativecommons.org/licenses/by/4.0/.

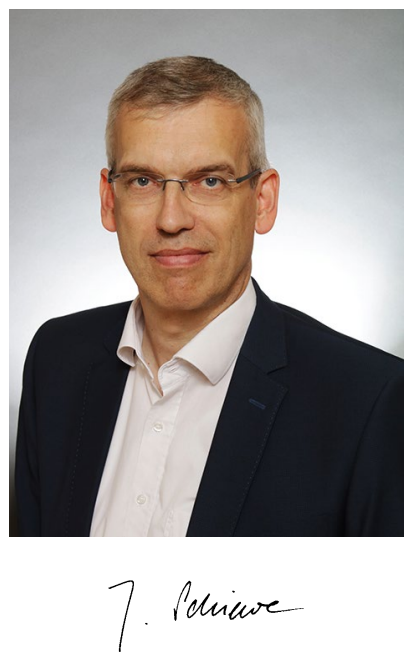

